\title{
Tetrolet Transform: A New Adaptive Haar Wavelet Algorithm for Sparse Image Representation
}

\author{
JENS KROMMWEH \\ Department of Mathematics, \\ University of Duisburg-Essen, Campus Duisburg, \\ 47048 Duisburg, Germany \\ jens.krommweh@uni-due.de
}

January 27, 2009

\begin{abstract}
In order to get an efficient image representation we introduce a new adaptive Haar wavelet transform, called Tetrolet Transform. Tetrolets are Haar-type wavelets whose supports are tetrominoes which are shapes made by connecting four equal-sized squares. The corresponding filter bank algorithm is simple but enormously effective. In every level of the filter bank algorithm we divide the low-pass image into $4 \times 4$ blocks. Then in each block we determine a local tetrolet basis which is adapted to the image geometry in this block. An analysis of the adaptivity costs leads to modified versions of our method. Numerical results show the strong efficiency of the tetrolet transform for image compression.
\end{abstract}

Mathematics Subject Classification 2000. 65T60, 42C40, 68U10, 94A08.

Key words. sparse representation, wavelet transform on adaptive sets, data compression, directional wavelets, Haar-type wavelets, locally orthonormal wavelet basis, tetromino tiling, image approximation.

\section{Introduction}

The main task in every kind of image processing is finding an efficient image representation that characterizes the significant image features in a compact form. Here, the $2 \mathrm{D}$ discrete wavelet transform (DWT) is one of the most important tools. Conventionally, the 2D DWT is a separable construction, based on the 1D wavelet transformation which is independently applied to the rows and columns of an image. Therefore, the horizontal and vertical directions are preferred, and the DWT fails to achieve optimal results with images that contain geometric structures in other directions.

In the last years a lot of methods have been proposed to improve the treatment of orientated geometric image structures. Curvelets [3], contourlets [7], shearlets [10], and directionlets [16] are wavelet systems with more directional sensitivity. 
Instead of choosing a priori a basis or a frame one may adapt the function system depending on the local image structures. Wedgelets [6] and bandelets [14] stand for this second class of image representation schemes which is a wide field of further research. Very recent approaches are the grouplets [13] or the easy path wavelet transform (EPWT) [15] which are based on an averaging in adaptive neighborhoods of data points. While in the grouplet context neighborhood is defined by an association field, the EPWT uses neighborhoods on a path through all data points. Another kind of promising adaptive approaches is the usage of directional lifting-based wavelets [4], [5].

In this paper, we introduce a new adaptive algorithm whose underlying idea is simple but enormously effective. The construction is similar to the idea of digital wedgelets [8] where Haar functions on wedge partitions are considered. We divide the image into $4 \times 4$ blocks, then we determine in each block a tetromino partition which is adapted to the image geometry in this block. Tetrominoes are shapes made by connecting four equal-sized squares, each joined together with at least one other square along an edge. Originally, tetrominoes were introduced by Golomb [9], and they became popular through the famous computer game classic 'Tetris' [1], [2]. On these geometric shapes we define Haar-type wavelets, called tetrolets, which form a local orthonormal basis. The corresponding filter bank algorithm decomposes an image into a compact representation. In order to obtain a compressed image, one can apply a suitable shrinkage procedure to the tetrolet coefficients and reconstruct the image.

It should be mentioned that the tetrolet transform is not confined to image processing. Quite the contrary, the method is very efficient for compression of real data arrays.

The paper is organized as follows. In Section 2 we present the rough idea of the tetrolet transformation, in Section 3 a detailed description of the corresponding adaptive algorithm is given. Then in the next section, we show the important fact that the tetrolets form a local orthonormal basis. Section 5 is devoted to the analysis of the adaptivity cost which results in modified versions of the tetrolet transform. Finally, in the last section we present some numerical results applying the tetrolet transform to image approximation.

\section{The Tetrolet Transform: A New Locally Adaptive Algorithm}

\subsection{Definitions and Notations}

In order to explain the idea of the tetrolet transform we first need some definitions and notations. For simplicity we restrict our considerations to two-dimensional square data sets. Let $I=$ $\{(i, j): i, j=0, \ldots, N-1\} \subset \mathbb{Z}^{2}$ be the index set of a digital image $\mathbf{a}=(a[i, j])_{(i, j) \in I}$ with $N=2^{J}, J \in \mathbb{N}$. We determine a 4 -neighborhood of an index $(i, j) \in I$ by

$$
N_{4}(i, j):=\{(i-1, j),(i+1, j),(i, j-1),(i, j+1)\} .
$$

An index that lies at the boundary has three neighbors, an index at the vertex of the image has two neighbors. If advantageous, we shall use for several analysis a one-dimensional index set $J(I)$ by taking the bijective mapping $J: I \rightarrow\left\{0,1, \ldots, N^{2}-1\right\}$ with $J((i, j)):=j N+i$. 


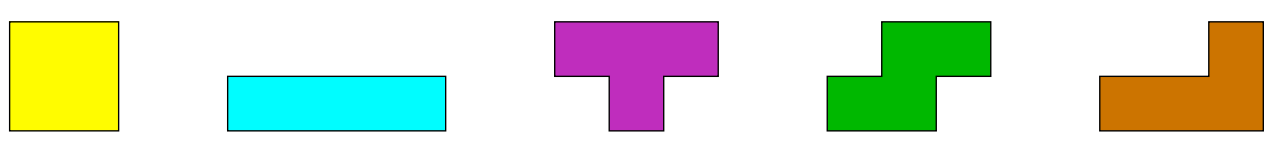

Figure 1: The five free tetrominoes. O-I-T-S-L-tetromino.

A set $E=\left\{I_{0}, \ldots, I_{r}\right\}, r \in \mathbb{N}$, of subsets $I_{\nu} \subset I$ is a disjoint partition of $I$ if $I_{\nu} \cap I_{\mu}=\emptyset$ for

$\nu \neq \mu$ and $\bigcup_{\nu=0}^{r} I_{\nu}=I$. In this paper we consider disjoint partitions $E$ of the index set $I$ that satisfy two conditions:

1. each subset $I_{\nu}$ contains four indices, i.e. $\# I_{\nu}=4$, and

2. every index of $I_{\nu}$ has a neighbor in $I_{\nu}$, i.e. $\forall(i, j) \in I_{\nu} \exists\left(i^{\prime}, j^{\prime}\right) \in I_{\nu}:\left(i^{\prime}, j^{\prime}\right) \in N_{4}(i, j)$.

We call such subsets $I_{\nu}$ tetromino, since the tiling problem of the square $[0, N)^{2}$ by shapes called tetrominoes [9] is a well-known problem being closely related to our partitions of the index set $I=\{0,1, \ldots, N-1\}^{2}$. We shortly recall this tetromino tiling problem in the next subsection.

For a simple one-dimensional indexing of the four elements in one tetromino subset $I_{\nu}$, we apply the bijective mapping $J$ as follows. For $I_{\nu}=\left\{\left(i_{1}, j_{1}\right),\left(i_{2}, j_{2}\right),\left(i_{3}, j_{3}\right),\left(i_{4}, j_{4}\right)\right\}$ let $L: I_{\nu} \rightarrow\{0,1,2,3\}$ be given by the rule that we order the values $J\left(i_{1}, j_{1}\right), \ldots, J\left(i_{4}, j_{4}\right)$ by size and map them to $\{0,1,2,3\}$ such that the smallest index is identified with 0 and the largest with 3.

\subsection{Tilings by Tetrominoes}

Tetrominoes were introduced by Golomb in [9]. They are shapes formed from a union of four unit squares, each connected by edges, not merely at their corners. The tiling problem with tetrominoes became popular through the famous computer game classic 'Tetris' [1], [2]. Disregarding rotations and reflections there are five different shapes, the so called free tetrominoes, see Figure 1.

Taking the isometries into account, it is clear that every square $[0, N)^{2}$ can be covered by tetrominoes if and only if $N$ is even. Already in 1937, Larsson showed that there are 117 solutions for disjoint covering of a $4 \times 4$ board with four tetrominoes [12]. For an $8 \times 8$ board we compute $117^{4}>10^{8}$ as a rough lower bound of possible tilings. Thus, in order to handle the number of solutions, it will be reasonable to restrict ourselves to an image partition into $4 \times 4$ squares.

As represented in Figure 2, we have 22 fundamental solutions in the $4 \times 4$ board (disregarding rotations and reflections). One solution (first line) is unaltered by rotations and reflections, four solutions (second line) give a second version applying the isometries. Seven forms can occur in four orientations (third line), and ten asymmetric cases in eight directions (last line). Further studies with tetrominoes can be found in [11]. 

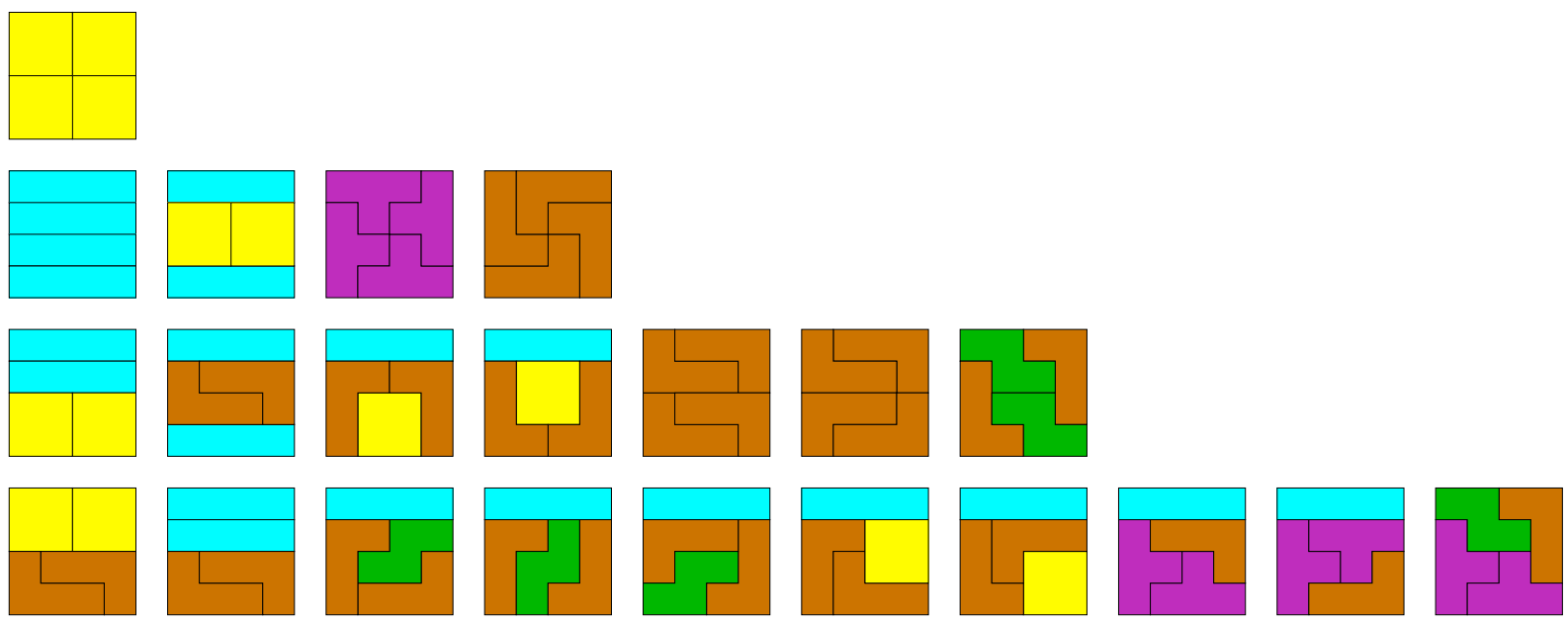

Figure 2: The 22 fundamental forms tiling a $4 \times 4$ board. Regarding additionally rotations and reflections there are 117 solutions.

\subsection{The Idea of Tetrolets}

The two-dimensional classical Haar wavelet decomposition leads to a special tetromino partition. Introducing the discrete tetrolet transformation, we recall the conventional Haar case in a notation consistent to the following tetrolet idea.

In the Haar filter bank, the low-pass filter and the high-pass filters are just given by the averaging sum and the averaging differences of each four pixel values which are arranged in a $2 \times 2$ square. More precisely, with $I_{i, j}=\{(2 i, 2 j),(2 i+1,2 j),(2 i, 2 j+1),(2 i+1,2 j+1)\}$ for $i, j=0,1, \ldots, \frac{N}{2}-1$, we have a dyadic partition $E=\left\{I_{0,0}, \ldots, I_{\frac{N}{2}-1, \frac{N}{2}-1}\right\}$ of the image index set $I$. Let $L$ be the bijective mapping mentioned above which maps the four pixel pairs of $I_{i, j}$ to the set $\{0,1,2,3\}$, i.e., it brings the pixels into a unique order.

Then we can determine the low-pass part

$$
\mathbf{a}^{1}=\left(a^{1}[i, j]\right)_{i, j=0}^{\frac{N}{2}-1} \quad \text { with } \quad a^{1}[i, j]=\sum_{\left(i^{\prime}, j^{\prime}\right) \in I_{i, j}} \epsilon\left[0, L\left(i^{\prime}, j^{\prime}\right)\right] a\left[i^{\prime}, j^{\prime}\right]
$$

as well as the three high-pass parts for $l=1,2,3$

$$
\mathbf{w}_{l}^{1}=\left(w_{l}^{1}[i, j]\right)_{i, j=0}^{\frac{N}{2}-1} \quad \text { with } \quad w_{l}^{1}[i, j]=\sum_{\left(i^{\prime}, j^{\prime}\right) \in I_{i, j}} \epsilon\left[l, L\left(i^{\prime}, j^{\prime}\right)\right] a\left[i^{\prime}, j^{\prime}\right],
$$

where the coefficients $\epsilon[l, m], l, m=0, \ldots, 3$, are entries from the Haar wavelet transform matrix

$$
W:=(\epsilon[l, m])_{l, m=0}^{3}=\frac{1}{2}\left(\begin{array}{cccc}
1 & 1 & 1 & 1 \\
1 & 1 & -1 & -1 \\
1 & -1 & 1 & -1 \\
1 & -1 & -1 & 1
\end{array}\right) .
$$

Obviously, the fixed blocking by the dyadic squares $I_{i, j}$ is very inefficient because the local structures of an image are disregarded. Our idea is to allow more general partitions such that 
the local image geometry is taken into account. Namely, we use tetromino partitions. As described in the previous subsection, we divide the image index set into $4 \times 4$ blocks. Then instead of using the classical Haar wavelet transform, which corresponds to a partition of the $4 \times 4$ block into squares (as in the first line of Figure 2), we compute the 'optimal' partition of the block into four tetrominoes according to the geometry of the image. In the following detailed description of the algorithm, we will explain more precisely what 'optimal' means, namely that the wavelet coefficients defined on the tetrominoes have minimal $l^{1}$-norm.

\section{Detailed Description of the Tetrolet Filter Bank Algorithm}

The rough structure of the tetrolet filter bank algorithm is described in Table 1 .

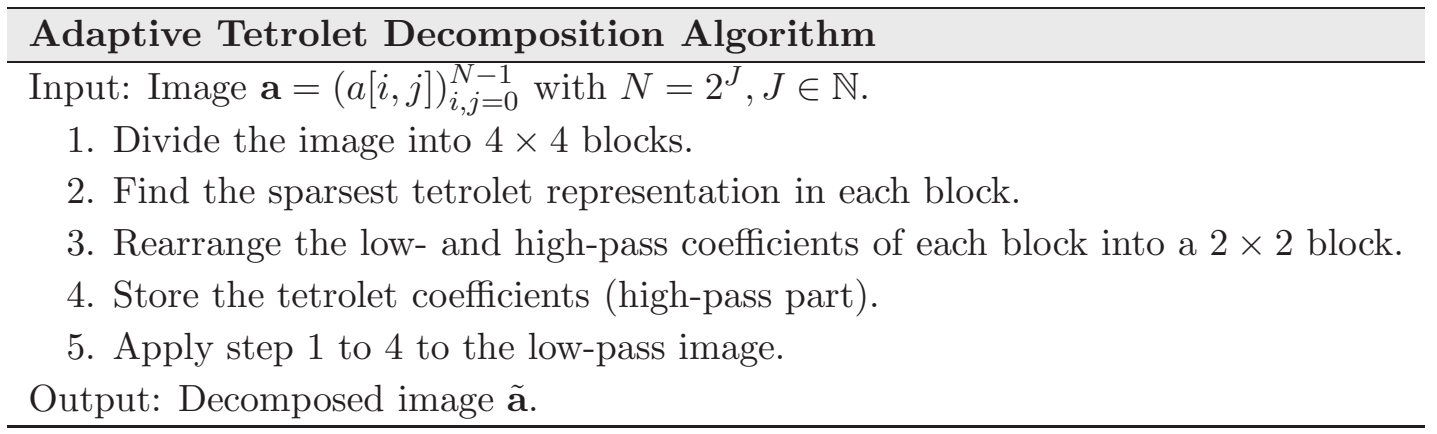

Table 1: Adaptive tetrolet decomposition algorithm.

Let us now go into detail considering separately each step of the tetrolet decomposition algorithm. Of course, our main attention shall be turned to step 2 of the algorithm. This is the step where the adaptivity comes into play.

We start with the input image $\mathbf{a}^{0}=(a[i, j])_{i, j=0}^{N-1}$ with $N=2^{J}, J \in \mathbb{N}$. Then we will be able to apply $J-1$ levels of the tetrolet transform. In the $r$ th-level, $r=1, \ldots, J-1$, we do the following computations.

1. Divide the low-pass image $\mathbf{a}^{r-1}$ into blocks $Q_{i, j}$ of size $4 \times 4, i, j=0, \ldots, \frac{N}{4^{r}}-1$.

2. In each block $Q_{i, j}$ we consider the 117 admissible tetromino coverings $c=1, \ldots, 117$. For each tiling $c$ we apply a Haar wavelet transform to the four tetromino subsets $I_{s}^{(c)}, s=$ $0,1,2,3$. In this way we obtain for each tiling $c$ four low-pass coefficients and 12 tetrolet coefficients. More precisely, in $Q_{i, j}$ we compute analogously to (2.1) and (2.2) the pixel averages for every admissible tetromino configuration $c=1, \ldots, 117$ by

$$
\mathbf{a}^{r,(c)}=\left(a^{r,(c)}[s]\right)_{s=0}^{3} \quad \text { with } \quad a^{r,(c)}[s]=\sum_{(m, n) \in I_{s}^{(c)}} \epsilon[0, L(m, n)] a^{r-1}[m, n],
$$

as well as the three high-pass parts for $l=1,2,3$

$$
\mathbf{w}_{l}^{r,(c)}=\left(w_{l}^{r,(c)}[s]\right)_{s=0}^{3} \quad \text { with } \quad w_{l}^{r,(c)}[s]=\sum_{(m, n) \in I_{s}^{(c)}} \epsilon[l, L(m, n)] a^{r-1}[m, n],
$$


where the coefficients $\epsilon[l, L(m, n)]$ are given in (2.3) and where $L$ is the bijective mapping mentioned in subsection 2.1 relating the four index pairs $(m, n)$ of $I_{s}^{(c)}$ with the values $0,1,2$, and 3 in descending order. That means, by the one-dimensional indexing $J(m, n)$ the smallest index is identified with the value 0 , while the largest with 3 .

Then we choose the covering $c^{*}$ such that the $l^{1}$-norm of the 12 tetrolet coefficients becomes minimal

$$
c^{*}=\arg \min _{c} \sum_{l=1}^{3}\left\|\mathbf{w}_{l}^{r,(c)}\right\|_{1}=\arg \min _{c} \sum_{l=1}^{3} \sum_{s=0}^{3}\left|w_{l}^{r,(c)}[s]\right| .
$$

Hence, for every block $Q_{i, j}$ we get an optimal tetrolet decomposition $\left[\mathbf{a}^{r,\left(c^{*}\right)}, \mathbf{w}_{1}^{r,\left(c^{*}\right)}, \mathbf{w}_{2}^{r,\left(c^{*}\right)}, \mathbf{w}_{3}^{r,\left(c^{*}\right)}\right]$. By doing this, the local structure of the image block is adapted. The best covering $c^{*}$ is a covering whose tetrominoes do not intersect an important structure like an edge in the image $\mathbf{a}^{r-1}$. Because the tetrolet coefficients become as minimal as possible a sparse image representation will be obtained. For each block $Q_{i, j}$ we have to store the covering $c^{*}$ that has been chosen, since this information is necessary for reconstruction. If the optimal covering is not unique, then we take the tiling $c^{*}$ that has already been chosen most frequently in the previous blocks. Thus, the coding of the used coverings becomes cheaper.

3. In order to be able to apply further levels of the tetrolet decomposition algorithm, we rearrange the entries of the vectors $\mathbf{a}^{r,\left(c^{*}\right)}$ and $\mathbf{w}_{l}^{r,\left(c^{*}\right)}$ into $2 \times 2$ matrices using a reshape function $R$,

$$
\left.\mathbf{a}_{\left.\right|_{Q_{i, j}} ^{r}}^{r}=R\left(\mathbf{a}^{r,\left(c^{*}\right)}\right)\right)=\left(\begin{array}{ll}
a^{r,\left(c^{*}\right)}[0] & a^{r,\left(c^{*}\right)[2]} \\
a^{r,\left(c^{*}\right)}[1] & a^{r,\left(c^{*}\right)[3]}
\end{array}\right),
$$

and in the same way $\mathbf{w}_{\left.l\right|_{Q_{i, j}}}^{r}=R\left(\mathbf{w}_{l}^{r,\left(c^{*}\right)}\right)$ for $l=1,2,3$, see Figure 3. For an efficient representation in the next level, a suitable arrangement of the low-pass values is essential. That means, the order of labeling the tetrominoes of $c^{*}$ in each block $Q_{i, j}$ by $s=0,1,2$, and 3 is very important. The labeling should be done in a way, such that the geometry of the tiling is suitably mapped to $\left(\begin{array}{ll}0 & 2 \\ 1 & 3\end{array}\right)$. Therefore we label the four shapes of the chosen partition $c^{*}$ by comparing with the square case. Among the 24 possibilities to label the four tetrominoes, the numbering with the highest correlation with the Haar partition is preferred. See an illustration in Figure 3: For comparison of different labeling of the four tetrominoes, we have computed the number of deviations from the Haar wavelet tiling, i.e. we count the number of small squares in a block $Q_{i, j}$, where the label differs from the label of these squares in the Haar wavelet tiling. We apply the order with minimal deviations. This optimal order needs not to be unique. The labeling in Figure 3(a) would lead to a distorted low-pass image, while 3(b) shows a reasonable order.

4. After finding a sparse representation in every block $Q_{i, j}$ for $i, j=0, \ldots, \frac{N}{4^{r}}-1$, we store (as usually done) the low-pass matrix $\mathbf{a}^{r}=\left(\mathbf{a}_{\left.\right|_{Q_{i, j}} ^{r}}\right)_{i, j=0}^{\frac{N}{4^{r}}-1}$ and the high-pass matrices $\mathbf{w}_{l}^{r}=$ 

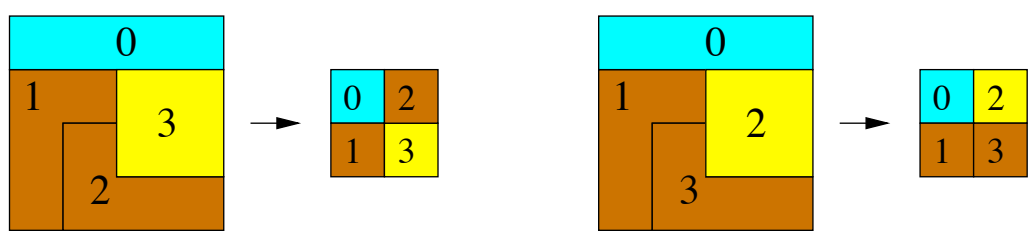

Figure 3: Example of labeling tetrominoes with corresponding low-pass image block. (a) Bad order (ten deviations from square case), (b) best order (eight deviations).

$$
\begin{gathered}
\left(\mathbf{w}_{\left.l\right|_{Q, j}}^{r}\right)_{i, j=0}^{\frac{N}{4^{r}}-1}, l=1,2,3 \text {, replacing the low-pass image } \mathbf{a}^{r-1} \text { by the matrix } \\
\qquad\left(\begin{array}{cc}
\mathbf{a}^{r} & \mathbf{w}_{2}^{r} \\
\mathbf{w}_{1}^{r} & \mathbf{w}_{3}^{r}
\end{array}\right) .
\end{gathered}
$$

After a suitable number of decomposition steps, we apply a shrinkage procedure to the tetrolet coefficients in order to get a sparse image representation. In our experiments in Section 6, we shall use the hard threshold function

$$
S_{\lambda}(x)= \begin{cases}x, & |x| \geq \lambda \\ 0, & |x|<\lambda .\end{cases}
$$

For the reconstruction of the image, we need the low-pass coefficients from the coarsest level and the tetrolet coefficients as usual. Additionally, the information about the respective covering in each level and block is necessary.

Remark 3.1. Note that we can understand the adaptive tetrolet filter bank as a Haar wavelet filter bank with locally permuted pixel values. This interpretation leads to an efficient implementation. More precisely, we put the 16 pixel values of each $4 \times 4$ image block $Q_{i, j}$ into a vector $\mathbf{q}=\left(q_{1}, \ldots, q_{16}\right)^{T}$ by stacking the columns of the image block into $\mathbf{q}$. After applying a permutation matrix $P_{c} \in \mathbb{R}^{16 \times 16}$, which rearranges the image values according to an optimal tetromino covering $c \in\{1, \ldots, 117\}$, we compute the low- and high-pass parts from (3.1) and (3.2) by

$$
\tilde{\mathbf{q}}=\left(I_{4} \otimes W\right) P_{c} \mathbf{q},
$$

where $W$ is the Haar transformation matrix from (2.3), $I_{4}$ is the unit matrix of size $4 \times 4$, and $\otimes$ is the Kronecker product, i.e. $\left(I_{4} \otimes W\right)=\operatorname{blockdiag}(W, W, W, W)$. The resulting vector $\tilde{\mathbf{q}} \in \mathbb{R}^{16}$ has the form

$$
\tilde{\mathbf{q}}=\left(a^{r,(c)}[0], w_{1}^{r,(c)}[0], w_{2}^{r,(c)}[0], w_{3}^{r,(c)}[0], \ldots, a^{r,(c)}[3], w_{1}^{r,(c)}[3], w_{2}^{r,(c)}[3], w_{3}^{r,(c)}[3]\right)^{T} .
$$



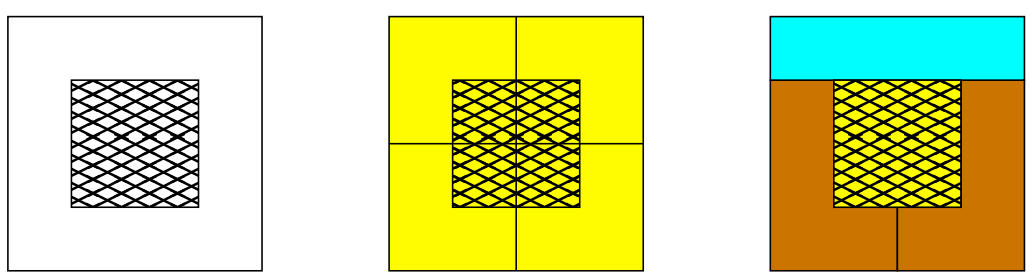

Figure 4: Example of block covering by adaptive tetrominoes. (a) image function, (b) square supports of classical Haar wavelets, (c) supports of adaptive tetrolets.

Example. Consider the $4 \times 4$ block of a synthetic image given by

$$
\left[\begin{array}{cccc}
20 & 20 & 20 & 20 \\
20 & 160 & 160 & 20 \\
20 & 160 & 160 & 20 \\
20 & 20 & 20 & 20
\end{array}\right]
$$

see Figure 4(a). The conventional Haar wavelet transform of the image function has maximal number of non zero wavelet coefficients. Using (2.1) and (2.2) we obtain after one decomposition step

$$
\left[\begin{array}{cc}
\mathbf{a}^{1} & \mathbf{w}_{2}^{1} \\
\mathbf{w}_{1}^{1} & \mathbf{w}_{3}^{1}
\end{array}\right]=\left[\begin{array}{cccc}
110 & 110 & -70 & -70 \\
110 & 110 & 70 & 70 \\
-70 & 70 & 70 & -70 \\
-70 & 70 & -70 & 70
\end{array}\right]
$$

All 12 wavelet coefficients do not vanish. By contrast, if we take the tetrolet transform, all pixel differences will vanish according to the adaptive covering by tetrominoes in Figure 4(c). Note, that regarding rotations there are four optimal partitions by tetrominoes.

We stack the columns of (3.5) into a vector

$$
\mathbf{q}=(20,20,20,20,20,160,160,20,20,160,160,20,20,20,20,20)^{T}
$$

and apply a permutation matrix $P_{c}$ corresponding to the tetromino covering in Figure 4(c). In our case, it means, that $P_{c}$ is given by

$$
P_{c}(1, \ldots, 16)^{T}=(1,5,9,13,2,3,4,8,6,7,10,11,12,14,15,16)^{T} .
$$

So, we get indeed a sparse representation $\tilde{\mathbf{q}}=(40,0,0,0,40,0,0,0,320,0,0,0,40,0,0,0)^{T}$ by (3.4) because all tetrolet coefficients are zero.

\section{An Orthonormal Basis of Tetrolets}

We describe the discrete basis functions which correspond to the above algorithm. Remember that the digital image $\mathbf{a}=(a[i, j])_{(i, j) \in I}$ is a subset of $l_{2}\left(\mathbb{Z}^{2}\right)$. For any tetromino $I_{\nu}$ of $I$ we define the discrete functions

$$
\phi_{I_{\nu}}[m, n]:=\left\{\begin{array}{cl}
1 / 2, & (m, n) \in I_{\nu}, \\
0, & \text { otherwise }
\end{array} \quad \text { and } \quad \psi_{I_{\nu}}^{l}[m, n]:=\left\{\begin{array}{cl}
\epsilon[l, L(m, n)], & (m, n) \in I_{\nu} \\
0, & \text { otherwise }
\end{array}\right.\right.
$$


Due to the underlying tetromino support, we call $\phi_{I_{\nu}}$ and $\psi_{I_{\nu}}^{l}$ tetrolets. As a straightforward consequence of the orthogonality of the standard 2D Haar basis functions and the partition of the discrete space by the tetromino supports, we have the following essential statement.

Theorem 4.1. For every admissible covering $\left\{I_{0}, I_{1}, I_{2}, I_{3}\right\}$ of a $4 \times 4$ square $Q \subset \mathbb{Z}^{2}$ the tetrolet system

$$
\left\{\phi_{I_{\nu}}: \nu=0,1,2,3\right\} \cup\left\{\psi_{I_{\nu}}^{l}: \nu=0,1,2,3 ; l=1,2,3\right\}
$$

is an orthonormal basis of $l^{2}(Q)$.

\section{Cost of Adaptivity: Modified Tetrolet Transform}

The tetrolet transform proposed in the previous sections reduces the number of wavelet coefficients compared with the classical tensor product wavelet transform. This improvement has to be payed with the storage of additional information which is not negligible. In this section we shall address this issue in detail. It will lead to some relaxed versions of the tetrolet transform in order to reduce the costs of adaptivity.

In the $r$ th decomposition level of the tetrolet transform we need to store $\frac{N^{2}}{4^{r+1}}$ covering values $c$, and therefore after $J$ levels one has $\frac{N^{2}}{12}\left(1-\frac{1}{4^{J}}\right)$ values. For a complete decomposition, i.e. for $J=\log _{2}(N)-1$ we have to store $\left(N^{2}-4\right) / 12$ values additionally.

It is well-known that a vector of length $N$ and with entropy $E$ can be stored with $N \cdot E$ bits, where the entropy

$$
E=-\sum_{i=1}^{n} p\left(x_{i}\right) \log _{2}\left(p\left(x_{i}\right)\right)
$$

describes the required bits per pixel (bpp) and is an appropriate measure for the quality of compression. The entropy (5.1) can be interpreted as the expected length of a binary code over all the symbols from the given alphabet $A=\left\{x_{1}, \ldots, x_{n}\right\}$. Here, $p\left(x_{i}\right)$ describes the relative occurrence of the symbol $x_{i}$ in the data set.

In the following, we propose three methods of entropy reduction in order to reduce the adaptivity costs. An application of these modified transforms as well as of combinations of them is given in the last section.

(a) The simplest approach of entropy reduction is a reduction of the alphabet $A$. The standard tetrolet transform selects the optimal covering in each image block from the alphabet $\{1, \ldots, 117\}$. Due to the similarity of some tetromino configurations (see Figure 2) we can disregard certain tilings. We restrict ourselves to 16 suitable configurations. Of course, the optimal choice of these configurations depends on the image. But we can choose a collection of tilings a priori by selecting configurations that feature different directions, see Figure 5. Furthermore, restricting to 16 configurations implicates an essential gain in computation time. 


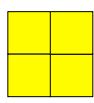

\begin{tabular}{ll|}
\hline & 0 \\
$\circ$ & $\circ$
\end{tabular}

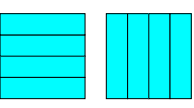

$\square$

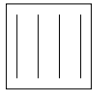

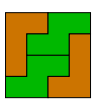
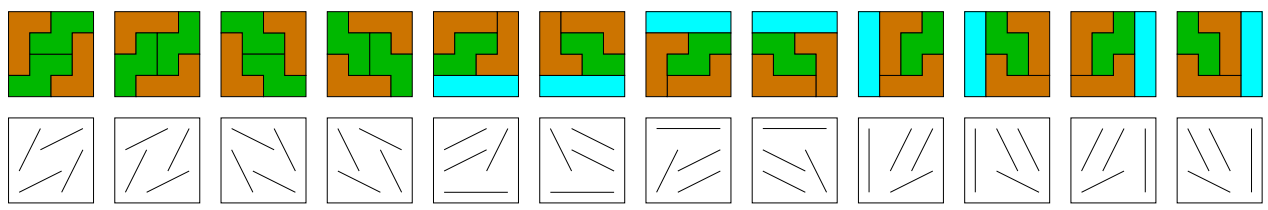

Figure 5: The a priori selected 16 tilings with different directions.

(b) A second approach to reduce the entropy is to manipulate the propabilities $p\left(x_{i}\right)$ in $(5.1)$ by changing their distribution. Relaxing the tetrolet transform we can demand that only very few tilings are preferred. Hence, we allow the choice of an almost optimal covering $c^{*}$ in (3.3) in order to get a tiling which is already frequently choosen. More precisely, we replace (3.3) by the two steps:

1. Find the set $A^{\prime} \subset A$ of almost optimal configurations $c$ that satisfy

$$
\sum_{l=1}^{3}\left\|\mathbf{w}_{l}^{r,(c)}\right\|_{1} \leq \min _{c \in A} \sum_{l=1}^{3}\left\|\mathbf{w}_{l}^{r,(c)}\right\|_{1}+\theta
$$

with a predetermined tolerance parameter $\theta$.

2. Among these tilings take the covering $c^{*} \in A^{\prime}$ which is chosen most frequently in the previous image blocks.

Using an appropriate relaxing parameter $\theta$, we achieve a satisfactory balance between low entropy (low adaptivity costs) and minimal tetrolet coefficients.

(c) The third method also reduces the entropy by optimization of the tiling distribution. After an application of an edge detector we use the classical Haar wavelet transform inside flat image regions. In image blocks that contain edges we make use of the strong adaptivity of the proposed tetrolet transform. This method leads to a huge amount of the square tiling corresponding to the Haar wavelet case.

\section{$6 \quad$ Numerical experiments}

As already mentioned in the beginning, our method is also very efficient for compression of real data arrays, but in the following we consider digital images.

While wavelet frames are useful for denoising (because redundancy information gives more hope to reconstruct the origin data), for image compression a basis is desirable. The tetrolets form a basis of a subspace of $l^{2}\left(\mathbb{Z}^{2}\right)$ and lead therefore to a sparse image representation using the efficient filter bank algorithm described above.

\section{Standard tetrolet transform}

We apply a complete wavelet decomposition of an image and use a wavelet shrinkage with global hard-thresholding choosing the threshold $\lambda$ such that a certain number of largest wavelet coefficients is retained. 
The $256 \times 256$ synthetic image in Figure 6 shows that the tetrolet transformation gives excellent results for piecewise constant images. With only 512 coefficients after thresholding, the reconstructed image achieves a remarkable PSNR of 38.47 because the orientated edges are well adapted. Though the Haar-like tetrolets are not continuous the Figures 7-10 illustrate that even for natural images the tetrolet filter bank outperforms the tensor product wavelets with the biorthogonal 9-7 filter bank. This confirms the fact already noticed with wedgelets [6] and bandlets [14]: While nonadaptive methods need smooth wavelets for excellent results, well constructed adaptive methods need not. For visual purposes the images are slightly smoothed with a bilateral filter in a postprocessing step.

The results are summarized in Table 2. The $256 \times 256$ images 'cameraman', 'pepper', and 'lena' were approximated with 2048 coefficients, the $64 \times 64$ detail monarch image with 256 coefficients. In particular, in the last example, Figure 10, the enormous efficiency in handling with several directional edges due to the high adaptivity can be well noticed.

\begin{tabular}{|l|c|c|c|c|c|}
\hline & $\begin{array}{c}\text { synthetic } \\
\text { (Fig. 6) }\end{array}$ & $\begin{array}{c}\text { cameraman } \\
\text { (Fig. 7) }\end{array}$ & $\begin{array}{c}\text { pepper } \\
\text { (Fig. 8) }\end{array}$ & $\begin{array}{c}\text { lena } \\
\text { (Fig. 9) }\end{array}$ & $\begin{array}{c}\text { monarch } \\
\text { (Fig. 10) }\end{array}$ \\
\hline Coefficients after shrinkage & 512 & 2048 & 2048 & 2048 & 256 \\
\hline Tensor Haar & 28.13 & 25.47 & 26.11 & 25.58 & 18.98 \\
Tensor biorthogonal 9-7 & 30.23 & 27.26 & 28.96 & 28.26 & 21.78 \\
Tetrolets & 38.47 & 29.17 & 30.00 & 28.98 & 24.43 \\
\hline
\end{tabular}

Table 2: PSNR values with approximation.

\section{Modified tetrolet transform}

Considering the adaptivity costs we compare the standard tetrolet transform and the modified versions. A complete tetrolet decomposition of the $256 \times 256$ cameraman image yields 5461 adaptivity values $c \in\{1, \ldots, 117\}$. The distribution of these values with the standard tetrolet transformation is shown in Figure 11(a); the entropy is $0.56 \mathrm{bpp}$. According to the second modification mentioned above we relax the tetrolet transform with $\theta=25$, the resulting histogram is given in Figure 11(b), the corresponding entropy is reduced to $0.25 \mathrm{bpp}$. This is a remarkable improvement under the small loss of quality (instead of 29.17 PSNR we have only 28.91 PSNR).

Of course, reduction of adaptivity cost produces a loss of approximation quality. Hence, a satisfactory balance is necessary. Table 3 presents some results applying the modified versions of the tetrolet transform proposed in the previous section to the monarch detail and the cameraman image. The three modifications (called 'Tetrolet 16', 'Tetrolet rel', 'Tetrolet edge') and a combination of them ('Tetrolet 16 rel edge') are compared with the tensor product wavelet transformation regarding to quality and storage costs. We have tried to balance the modified tetrolet transform such that the full costs are in the same scale as with the 9-7 filter. Then, one can observe that the tetrolets lead to slightly higher PSNR values than the 9-7 filter. For the relaxed versions we have used the global relaxation parameter $\theta=25$.

For a rough estimation of the complete storage costs of the compressed image with $N^{2}$ pixels 

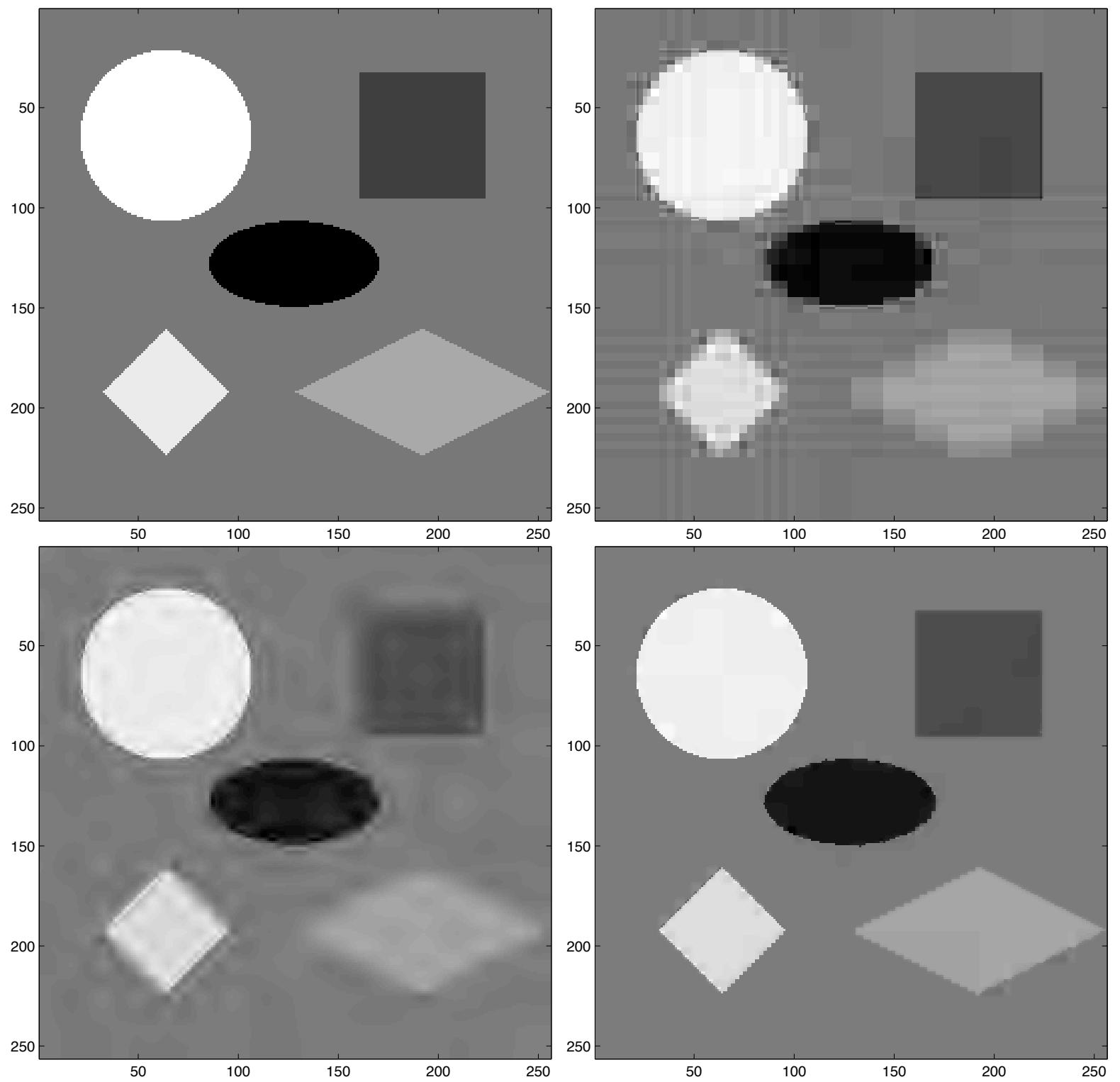

Figure 6: Approximation of a synthetic image with 512 coefficients. (a) Input, (b) classical Haar, (c) Biorthogonal 9-7, (d) Tetrolets.

we apply a simplified scheme

$$
\operatorname{cost}_{f u l l}=\operatorname{cost}_{W}+\operatorname{cost}_{P}+\operatorname{cost}_{A},
$$

where $\operatorname{cost}_{W}=16 \cdot M / N^{2}$ are the costs in bpp of storing $M$ non-zero wavelet coefficients with 16 bits. The term cost $_{P}$ gives the cost for coding the position of these $M$ coefficients by $-\frac{M}{N^{2}} \log _{2}\left(\frac{M}{N^{2}}\right)-\frac{N^{2}-M}{N^{2}} \log _{2}\left(\frac{N^{2}-M}{N^{2}}\right)$. The third component appearing only with the tetrolet transform contains the cost of adaptivity, $\operatorname{cost}_{A}=E \cdot R / N^{2}$, for $R$ adaptivity values and the entropy $E$ previously discussed. 

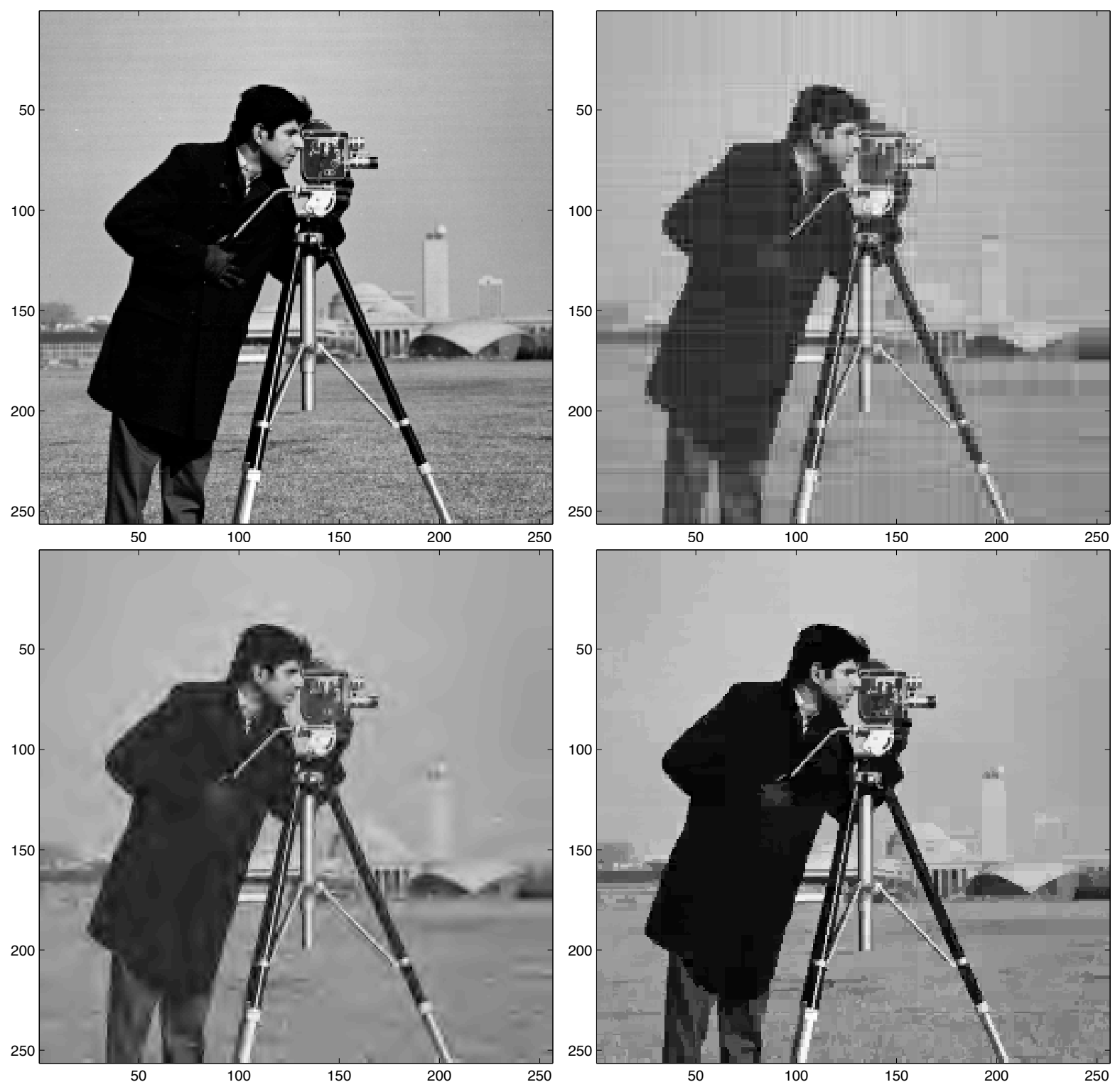

Figure 7: Approximation of the 'cameraman' image with 2048 coefficients. (a) Input, (b) classical Haar, (c) Biorthogonal 9-7, (d) Tetrolets.

\section{Acknowledgment}

The research in this paper is funded by the project PL 170/11-1 of the Deutsche Forschungsgemeinschaft (DFG). This is gratefully acknowledged. Furthermore the author would like to thank his advisor Gerlind Plonka for her helpful instructions and permanent support as well as Stefanie Tenorth and Michael Wozniczka for valuable comments. 

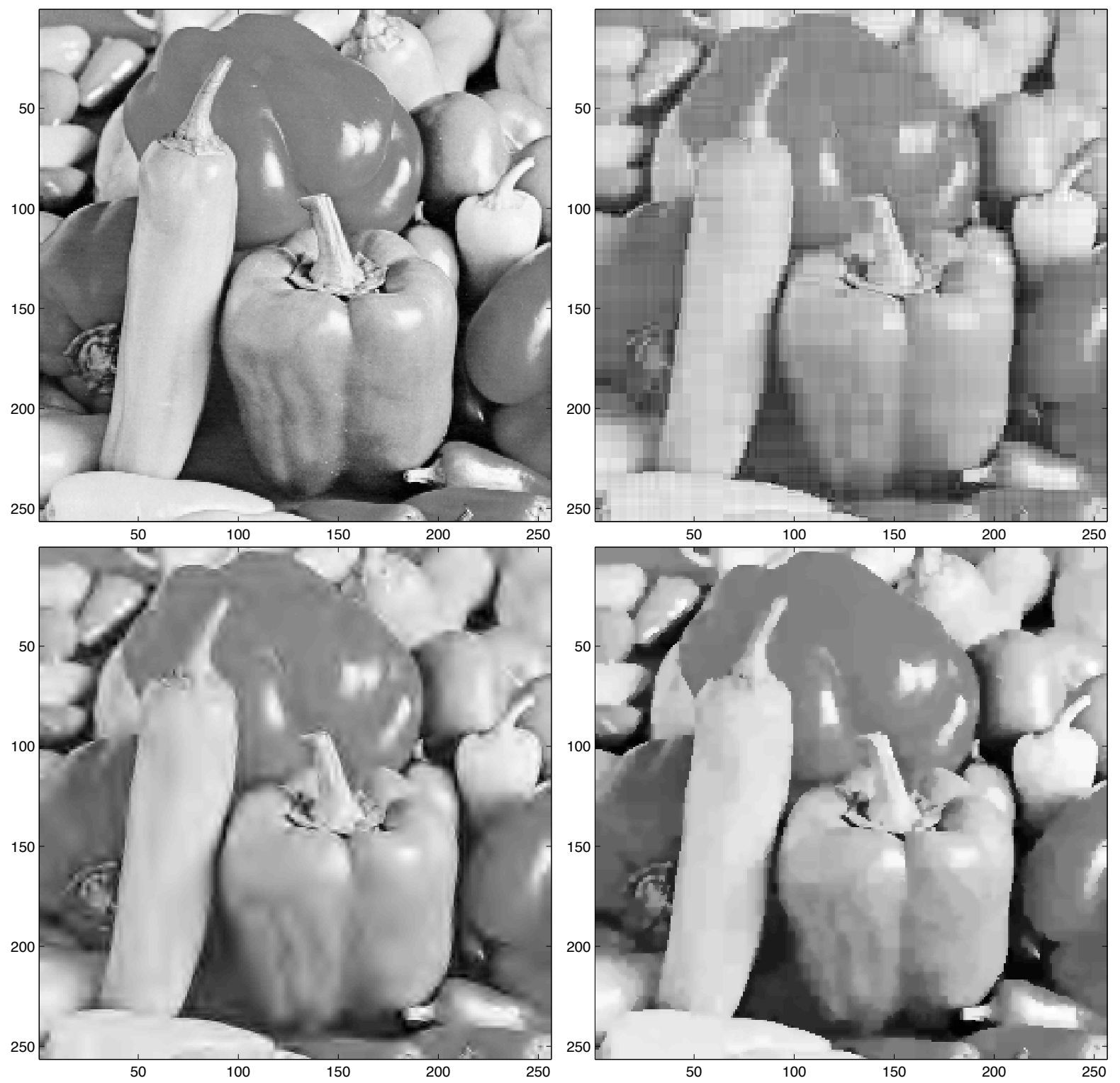

Figure 8: Approximation of the 'pepper' image with 2048 coefficients. (a) Input, (b) classical Haar, (c) Biorthogonal 9-7, (d) Tetrolets.

\section{References}

[1] R. Breukelaar, E. Demaine, S. Hohenberger, H. Hoogeboom, W. Kosters, and D. LibenNowell, Tetris is hard, even to approximate, International Journal of Computational Geometry and Applications 14 (2004), 41-68.

[2] R. Breukelaar, H. Hoogeboom, and W. Kosters. Tetris is hard, made easy, Technical Report 2003-9, Leiden Institute of Advanced Computer Science, 2003. 

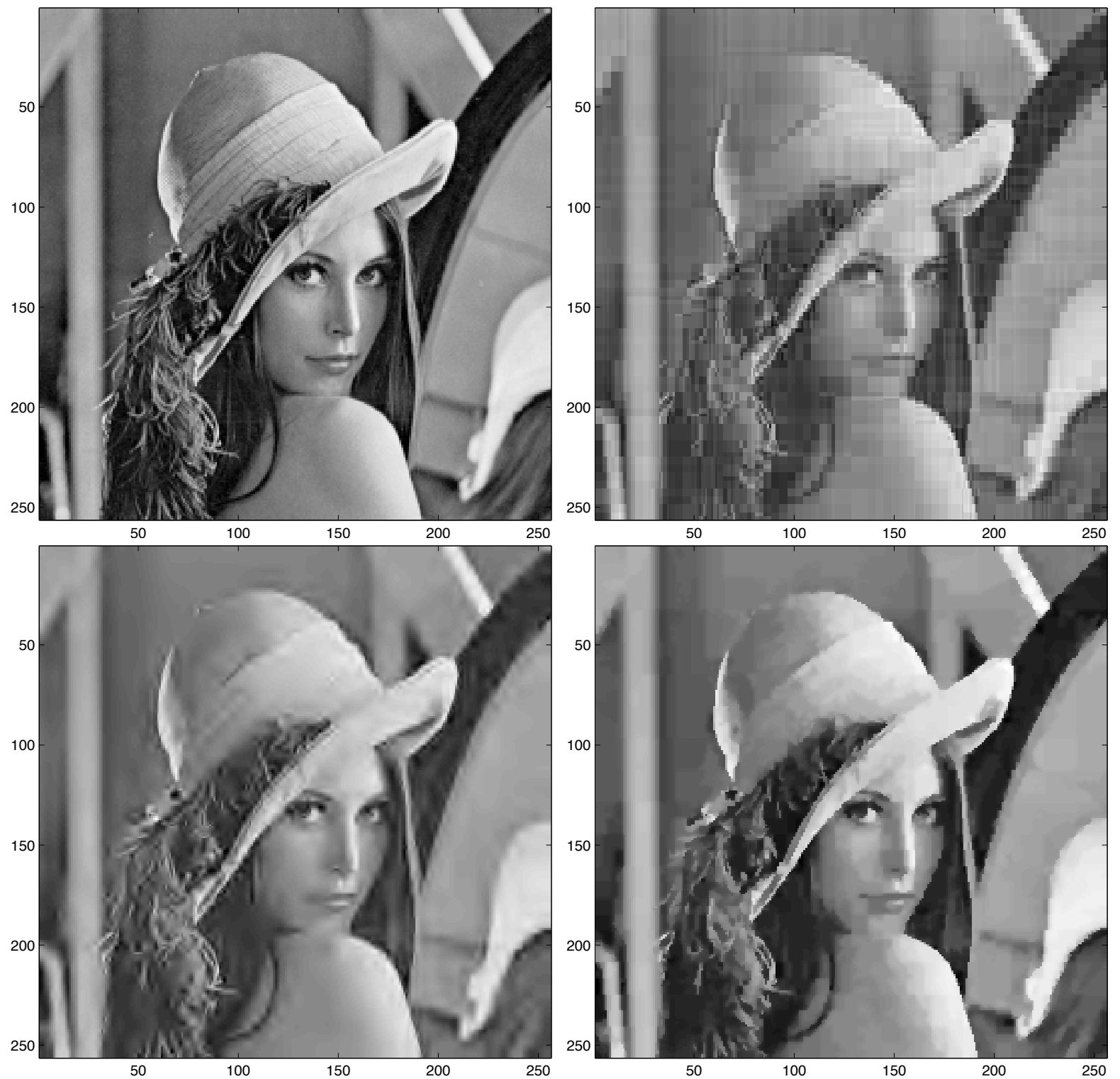

Figure 9: Approximation of the 'lena' image with 2048 coefficients. (a) Input, (b) classical Haar, (c) Biorthogonal 9-7, (d) Tetrolets.

[3] E.J. Candès and D.L. Donoho, New tight frames of curvelets and optimal representations of objects with piecewise $C^{2}$ singularities, Comm. Pure and Appl. Math. 56 (2004), 216-266.

[4] C.-L. Chang and B. Girod, Direction-adaptive discrete wavelet transform for image compression, IEEE Trans. Image Process. 16(5) (2007), 1289-1302.

[5] W. Ding, F. Wu, X. Wu, S. Li, and H. Li, Adaptive directional lifting-based wavelet transform for image coding, IEEE Trans. Image Process. 16(2) (2007), 416-427. 

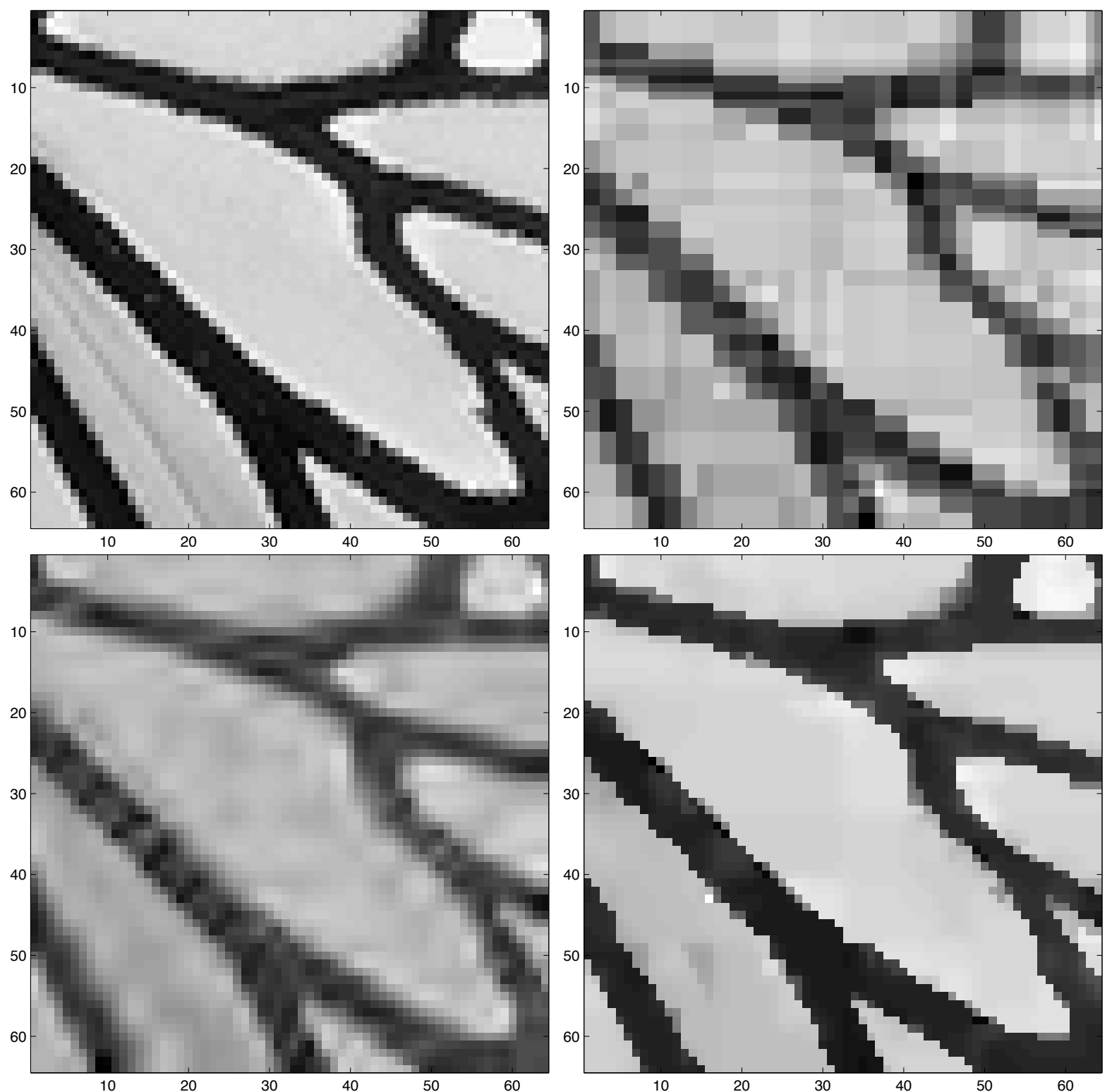

Figure 10: Approximation of the $64 \times 64$ detail image of 'monarch' with 256 coefficients. (a) Input, (b) classical Haar, (c) Biorthogonal 9-7, (d) Tetrolets.

[6] D.L. Donoho, Wedgelets: nearly minimax estimation of edges, Ann. Statist. 27(3), (1999), 859-897.

[7] M.N. Do and M. Vetterli, The contourlet transform: an efficient directional multiresolution image representation, IEEE Trans. Image Process. 14(12) (2005), 2091-2106.

[8] H. Führ, L. Demaret, and F. Friedrich, Beyond wavelets: New image representation paradigms. Book chapter, in: M. Barni and F. Bartolini, Document and Image Compression, Taylor and Francis, 2006. 

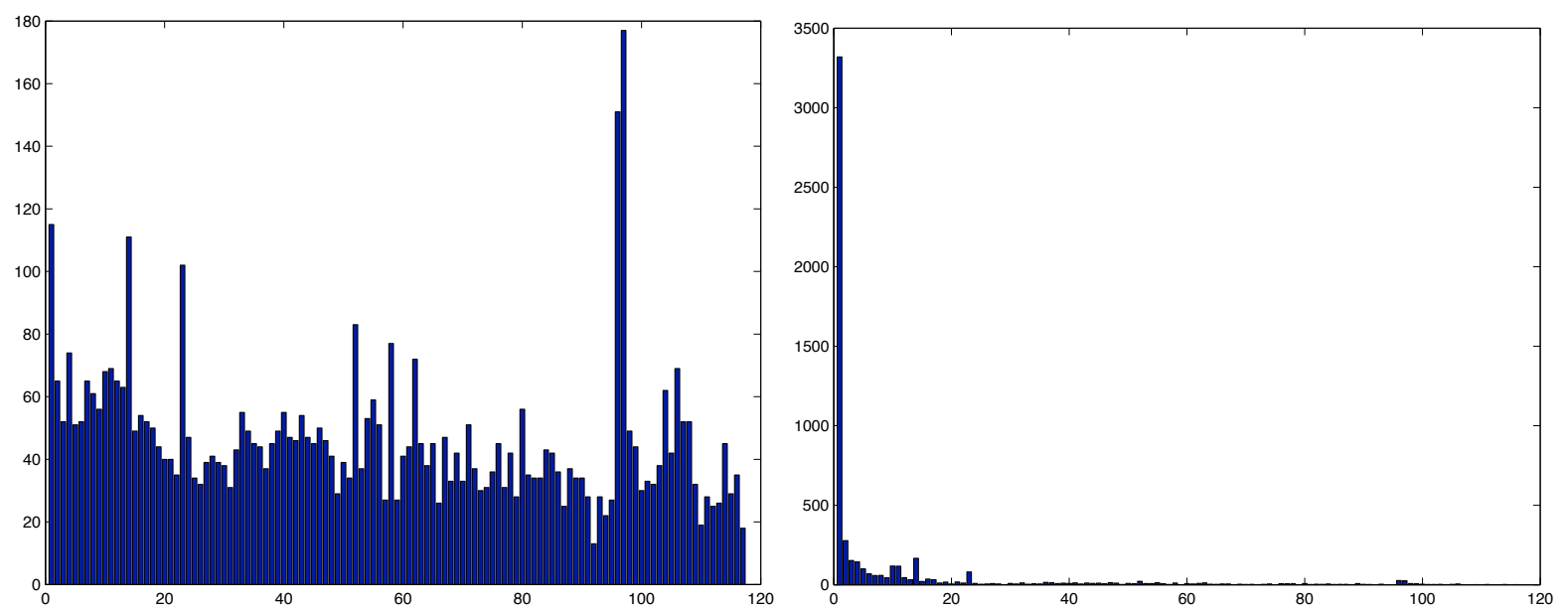

Figure 11: Distribution of the 5461 adaptivity values with the cameraman image. (a) Standard tetrolet transfrom, entropy is $0.56 \mathrm{bpp}$, (b) relaxed tetrolet transform with $\theta=25$, entropy is $0.25 \mathrm{bpp}$.

\begin{tabular}{|c|c|c|c|c|c|c|c|c|}
\hline & \multicolumn{4}{|c|}{ monarch } & \multicolumn{4}{|c|}{ cameraman } \\
\hline & coeff & PSNR & entropy & cost $_{\text {full }}$ & coeff & PSNR & entropy & cost $_{\text {full }}$ \\
\hline Tensor Haar & 300 & 19.58 & - & 1.55 & 2500 & 26.29 & - & 0.84 \\
\hline Tensor 9-7 & 300 & 22.62 & - & 1.55 & 2500 & 28.14 & - & 0.84 \\
\hline Tetrolet & 256 & 24.43 & 0.53 & 1.86 & 2048 & 29.17 & 0.56 & 1.26 \\
\hline Tetrolet 16 & 256 & 23.56 & 0.30 & 1.64 & 2048 & 28.44 & 0.32 & 1.02 \\
\hline Tetrolet rel & 256 & 24.51 & 0.32 & 1.66 & 2048 & 28.91 & 0.25 & 0.95 \\
\hline Tetrolet edge & 256 & 24.24 & 0.43 & 1.77 & 2048 & 28.94 & 0.32 & 1.02 \\
\hline Tetrolet 16 rel edge & 256 & 23.48 & 0.21 & 1.55 & 2048 & 28.24 & 0.14 & 0.84 \\
\hline
\end{tabular}

Table 3: Comparison between tensor wavelet transforms and different versions of the tetrolet transform regarding quality (PSNR) and storage cost ( cost $_{\text {full }}$ in bpp).

[9] S. W. Golomb, Polyominoes, Princeton University Press, 1994.

[10] K. Guo and D. Labate, Optimally sparse multidimensional representation using shearlets, SIAM J. Math. Anal. 39 (2007), 298-318.

[11] M. Korn, Geometric and algebraic properties of polyomino tilings, PhD thesis, Massachusetts Institute of Technology (2004).

[12] B. Larsson, Problem 2623, in: Fairy Chess Review 3(5) (1937), 51.

[13] S. Mallat, Geometrical grouplets, Appl. Comput. Harmon. Anal., to appear.

[14] E. Le Pennec and S. Mallat, Sparse geometric image representations with bandelets, IEEE Trans. Image Process. 14(4) (2005), 423-438. 
[15] G. Plonka, Easy path wavelet transform: a new adaptive wavelet transform for sparse representation of two-dimensional data, Multiscale Model. Simul., 2009, to appear.

[16] V. Velisavljević, B. Beferull-Lozano, M. Vetterli, and P.L. Dragotti, Directionlets: anisotropic multi-directional representation with separable filtering, IEEE Trans. Image Process. 17(7) (2006), 1916-1933. 